\title{
Humanitäre Hilfe vor grossen Herausforderungen
}

\section{Jean Martin}

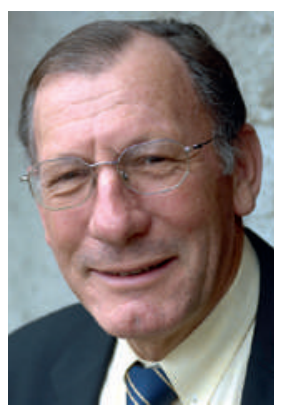

In der Regel lädt die Policlinique médicale universitaire (PMU) Lausanne immer einen aussergewöhnlichen Redner zu ihrer Jahresabschlussfeier. So waren bei diesem Anlass vor einigen Jahren Ella Maillart und Nicolas Bouvier zu hören. Im vergangenen Dezember hiess der Vortragende Yves Daccord, Generaldirektor des Internationalen Komitees vom Roten Kreuz (IKRK), das 13000 professionelle Mitarbeitende in 80 Ländern beschäftigt - mit steigender Tendenz.

Redner und Zuhörerschaft verbindet das gemeinsame Interesse an der Gesundheit. Mehrere Mitarbeitende der PMU waren bereits im humanitären Bereich engagiert. Der ausgezeichnete Vortrag analysiert die extreme Komplexität der heutigen Welt (die nichts mehr mit jener zu tun hat, in die junge Idealisten - darunter auch ich - noch vor einigen Jahrzehnten aufbrachen, um der Dritten Welt ihre «Lösungen» zu bringen). Die Problemstellungen von heute sind multipel und mehrdimensional. Probleme mit der Gesundheit, der Wasserversorgung, dem Wiederaufbau können nicht isoliert gesehen werden, alles ist miteinander verwoben, Mangel, Gefahren und Dringlichkeiten überlagern und potenzieren sich (siehe Haiti nach dem Erdbeben).

Auch die grossen, sich gegenüberstehenden Meinungsund Machtblöcke sind unterschiedlich. Früher war es der Kampf zwischen konservativ und progressiv. Heute ist dieser Zwiespalt nur einer unter vielen und wird erweitert um religiöse, ethnische, wirtschaftliche, klimatische und andere Dimensionen.

Hinzu kommt, dass ein bestimmter, bislang von der westlichen Welt vorgegebener Regelrahmen sein Ende findet. Wir können nicht mehr davon ausgehen, dass hohe Werte (wie Respektieren des anderen, seiner Rechte, Respektieren von Verfahrensweisen) «automatisch" anerkannt werden. Es herrscht ein Abbau des "sozialen Gefüges», das uns zusammenhielt, sagt der IKRK-Chef. Auf internationaler Ebene konstatiert er eine völlige Abwesenheit von politischer Konvergenz. Jene Mächte - die USA, bis zu einem gewissen Grad China, Russland oder Europa -, die diese Konvergenz bislang einfordern konnten, können dies nicht mehr. Selbst wenn sie sich darauf einigen können, in Bezug auf einen bestimmten Problempunkt Druck auszuüben, sind ihre Interessen in anderen Konfliktzonen entgegengesetzt - und letztlich passiert nichts (zudem wird der Einfluss des US-Präsidenten durch innenpolitische Querelen unterminiert). Es gibt keine grossen Führungspersönlichkeiten mehr, sagt Daccord, keinen Mandela, Che Guevara oder Simon Bolivar. Selbst die Mächtigsten unserer Welt sind in Bedrängnis, unsicher über den richtigen Weg, polarisiert durch die Fokussierung auf das Unmittelbare. Es gibt tiefgreifende Veränderungen bei den alten Gleichgewichten, aber niemand weiss, welches das neue ist.

Hinzu kommt, dass unsere neuen Kommunikationsmittel alles verändern. Mobiltelefone sind universell. Es gibt sie in den ärmsten Ländern. Die Bäuerinnen auf einem Dorfmarkt zahlen ihre Käufe per Smartphone. Diese Unmittelbarkeit stellt alles, auch das IKRK, unter mehr Alltagsdruck. Daccord nennt das Beispiel eines Einsatzes am Horn von Afrika, wo die Hilfe wegen logistischer Probleme mit viertägiger Verspätung eintraf und die Mitarbeitenden auf Unzufriedenheit vor Ort stiessen, da die Betroffenen über ihr iPhone erfahren hatten, dass zuvor die Hilfe in Asien in zwei Tagen angekommen und das mitgelieferte Feldlazarett besser ausgestattet war ...

Die Welt ist stark fragmentiert und gleichzeitig unglaublich vernetzt. Es fehlen jedoch anerkannte Autoritäten, funktionierende Strukturen und Rahmen. Stattdessen kommt es zu immer vielfältigeren Einflüssen. Der «Islamische Staat IS» kontrolliert Gebiete mit 10 Millionen Einwohnern.

Dennoch bleibt das IKRK ein wichtiger Ansprechpartner, häufig der einzige mit Zugang zu allen Konfliktparteien. Allerdings wird seine Arbeit noch durch eine weitere Tendenz erschwert: Vertrauen ist nicht mehr in Mode. Misstrauen und Lügen beherrschen mehr denn je internationale Turbulenzen. Trotz der ausserordentlichen Leistungen des IKRK ist man weniger bereit, seine in den Genfer Konventionen verbriefte Rolle anzuerkennen Verlangt wird, dass die Organisation ihre Unparteilichkeit nachweist. Dadurch wird - obwohl dies nur ein Aspekt unter vielen ist, aber dennoch ein wichtiger - die Arbeit vor Ort riskanter und die Sicherheit noch fragiler. Die in der PMU anwesenden Zuhörer von Yves Daccord pflegen und unterstützen oft minderbemittelte Patienten bei uns. Das ist ihre Arbeit und sie sind gut darin Die Herausforderungen und Dramen, mit denen sich die Mitarbeitenden des IKRK konfrontiert sehen, setzen einen anderen Massstab. 